\title{
Introduction to the Blockchain Engineering Minitrack
}

\author{
Hong-Mei Chen \\ University of Hawaii at Manoa, USA \\ hmchen@hawaii.edu
}

\author{
Hoh Peter In \\ Korea University, Seoul, South Korea \\ hoh_in@korea.ac.kr
}

This is the first year of the "Blockchain Engineering" minitrack. This minitrack is a timely forum because the global interest in blockchain has rapidly increased beyond cryptocurrencies and "enterprise blockchain" is expected to become a mainstream in few years. Since the Bitcoin launch in 2008, the number of cryptocurrencies available over the Internet has been growing: as of 19 August 2018, it is over 1600. Gartner's latest technology hype cycle puts blockchain past the peak of expectations. Going beyond mechanisms for cryptocurrencies, such as Bitcoin and Ethereum, big corporations are setting up blockchain consortia such as Hyperledger, Enterprise Ethereum Alliance (EEA), and R3, to focus on broader implications of blockchain and to evolve blockchain into "enterprise grade" technology. Numerous startups are developing blockchain-enabled applications. In the meantime, major enterprises are eager to edge their technology toward production, as a result, many pilot projects in enterprises are also being rapidly developed.

Blockchain indeed represents a paradigm shift from centralized computing to decentralized. The intense interests in blockchain center around the decentralized system's ability to provide transparency, security, (pseudo-) anonymity and data integrity without any third-party organization in control of the transactions; it thus can enable trustless transactions and disintermediated society. The idea that distributed applications can also be run in a decentralized way (called Dapps- Decentralized applications) has opened the door to a large realm of possibilities. In addition, blockchain-based decentralized architectures of databases, operating systems, storage services, and machine learning are being rapidly proposed, such as BigChainDB, EOS, FileCoin, and AI-Coin, as an alternative solution to our current centralized server or cloud-based architectures.

This minitrack aims at providing a forum for addressing the challenges arising from the paradigm shift and the "how to" of engineering a blockchain system for enterprises that can fundamentally change how business value is created, discovered, and realized. Different from other minitracks that mainly focus on the use cases of blockchain technology, the main focus of this minitrack is on the design and engineering issues of enterprise blockchain systems. We are very excited to present three papers that precisely fit the bill.

The first paper is entitled "Pushing softwaredefined blockchain components onto edge hosts" by Mayra Samaniego and Ralph Deters. This research introduces the novel idea of encapsulating the features of blockchain into software-defined components to distribute them towards edge devices of an IoT network. This work encapsulates three features of blockchain into software-defined components: smart contract, consensus, and in-chain data. These software-defined blockchain components are customized to fulfill the requirements of IoT networks. The results of evaluations show that the blockchain components perform well working separated but collaborating and supporting each other to get a blockchain implementation at the edge of the IoT network. This research shifts the focus towards edgecentric IoT implementations of cooperative blockchain components. This focus allows the integration of private blockchain solutions in IoT.

The second paper is entitled "Design of a forgetting blockchain: A possible way to accomplish GDPR compatibility" by Simon Farshid, Andreas Reitz, and Peter Roßbach. This research aims at addressing a critical blockchain issue for enterprise applications. One key property of the blockchain technology is its ability to secure old data against modification. This makes blockchain an append-only structure, where new data can be added but never removed. This is due to transaction immutability enabled by keeping a history of all transactions. Nevertheless, there are scenarios where it is necessary to delete submitted data from the chain after it is no longer needed. This becomes even more crucial with the introduction of the European General Data Protection Regulation (GDPR), a strict privacy law. European consumers can demand that their data be "forgotten" or deleted, posing a challenge for blockchain applications. Even when personal information is not directly put on the blockchain, historical data can be analyzed to reveal identities of pseudonyms. This research 
presents a prototype of a trust-free solution, i.e., a decentralized blockchain that "forgets".

The third paper is entitled "What does not fit can be made to fit! Trade-offs in distributed ledger technology designs," by Niclas, Kannengießer, Sebastian Lins, Tobias Dehling, and Ali Sunyaev. Distributed ledger technology (DLT), including blockchain, enables secure processing of transactions between untrustworthy parties in a decentralized system. However, DLT is available in different designs that exhibit diverse characteristics. Moreover, DLT characteristics have complementary and conflicting interdependencies. Hence, there will never be an ideal DLT design for all DLT use cases; instead, DLT implementations need to be configured to contextual requirements. Successful DLT configuration requires, however, a sound understanding of DLT characteristics and their interdependencies. This paper reviews DLT characteristics and organizes them into six groups. Furthermore, it condenses interdependencies of DLT characteristics into trade-offs that should be considered for successful deployment of DLT. This research results in DLT archetypes for common design objectives, such as security, usability, or performance. 